\title{
NOUVELle
}

\section{Les cellules souches \\ humaines sont capables \\ de néo-biosynthétiser des \\ nanoparticules magnétiques \\ après avoir dégradé des nanoparticules synthétiques}

Aurore Van de Walle ${ }^{1}$, Ali Abou-Hassan ${ }^{2}$, Nathalie Luciani ${ }^{1}$, Claire Wilhelm ${ }^{1}$
${ }^{1}$ Laboratoire matière et systèmes complexes, CNRS UMR 7057, Université Paris Diderot, 10 rue Alice Domon et Léonie Duquet, 75205 Paris Cedex 13, France.

${ }^{2}$ Sorbonne Université, CNRS, Physico-chimie des électrolytes et nanosystèmes interfaciaux, PHENIX, 75005 Paris, France. aurore.vandewalle@gmail.com claire.wilhelm@univ-paris-diderot.fr
> Les nanoparticules magnétiques, qui contiennent du fer, sont au cœur de la nanomédecine; elles ont des applications multiples dans divers domaines allant de l'imagerie à la médecine régénérative. Ces applications nécessitent généralement la pénétration des nanoparticules dans l'organisme, et plus particulièrement dans des cellules. La question de leur devenir à long terme dans cet environnement vivant est donc essentielle. Les résultats récents et assez inattendus que nous avons obtenus en utilisant des cellules souches humaines donnent quelques clés quant à ce devenir [1]. Ces cellules sont en effet capables, non seulement de dégrader et d'assimiler les nanoparticules par les voies du métabolisme du fer, mais également de synthétiser des nanoparticules de novo. La présence de nanoparticules magnétiques biogéniques avait déjà été observée chez l'homme, mais leur biosynthèse n'avait jamais été démontrée jusqu’à présent.

\section{Le contexte}

Les nanoparticules magnétiques sont utilisées en nanomédecine pour le diagnostic en imagerie par résonance magnétique nucléaire [2], comme agents anticancéreux par hyperthermie [3] ou pour le ciblage de médicaments [4], ou encore comme agents pour la fabrication d'assemblages cellulaires en ingénierie tissulaire [5]. Mais que deviennent les nanoparticules magnétiques après avoir accompli leur mission diagnostique ou thérapeutique? Cette question est indissociable de celle du développement de leurs applications biomédicales afin d'en garantir la sécurité, et s'inscrit dans une réflexion bioéthique nécessaire sur le devenir de ces nanoparticules. L'attention portée au «cycle de vie » et à l'évolution à long terme des nanomatériaux est pourtant relativement nouvelle dans le domaine de la nanomédecine, qui s'est jusqu'à présent davantage focalisée sur la multi-fonctionnalité des nanoparticules et leur efficacité à court terme que sur leur devenir à long terme.

\section{L'objectif}

Nous avons étudié le devenir de nanoparticules magnétiques synthétiques pendant plusieurs semaines après leur incorporation dans des cellules souches, en utilisant le magnétisme des cellules comme un marqueur de la présence et de l'intégrité des nanoparticules [1]. Afin de déterminer l'influence de la différenciation cellulaire sur le devenir intracellulaire de ces nanoparticules, nous avons utilisé différentes conditions de culture qui favorisent la différenciation des cellules souches dans la voie de la chondrogenèse (transformation en chondrocytes), ou celle de l'adipogenèse (adipocytes) ou de l'ostéogenèse (ostéocytes), ou pour simplement maintenir ces cellules dans leur état indifférencié [1].
Le devenir des nanoparticules suivi par la mesure du magnétisme des cellules Au cours de cette étude, nous avons utilisé une approche originale de nanomagnétisme en milieu vivant: les nanoparticules sont tout d'abord incorporées dans des cellules souches humaines (Figure l), qui sont ensuite soumises à différents environnements, en culture 2D (monocouche de cellules avec conditions de maintien dans un état indifférencié, ou bien conditions de différenciation en adipocytes ou en ostéocytes), ou en culture 3D (formation de sphéroïdes pour la différenciation en chondrocytes). Ces modèles tissulaires permettent d'étudier le comportement et le devenir des nanoparticules sur le long terme dans différents environnements intracellulaires. Le magnétisme est alors mesuré sur une population de 200000 cellules, et sa variation reflète directement les processus de transformation des nanoparticules.

Un résultat surprenant: une néo-synthèse de nanoparticules magnétiques après leur dégradation Dans le modèle de culture 3D en sphéroïdes (condition de la chondrogenèse), l'aimantation des cellules décroît de manière importante pendant les 10 premiers jours qui suivent l'incorporation des nanoparticules (Figure 2A) : les nanoparticules sont dégradées en perdant leur structure cristalline, ce qui 


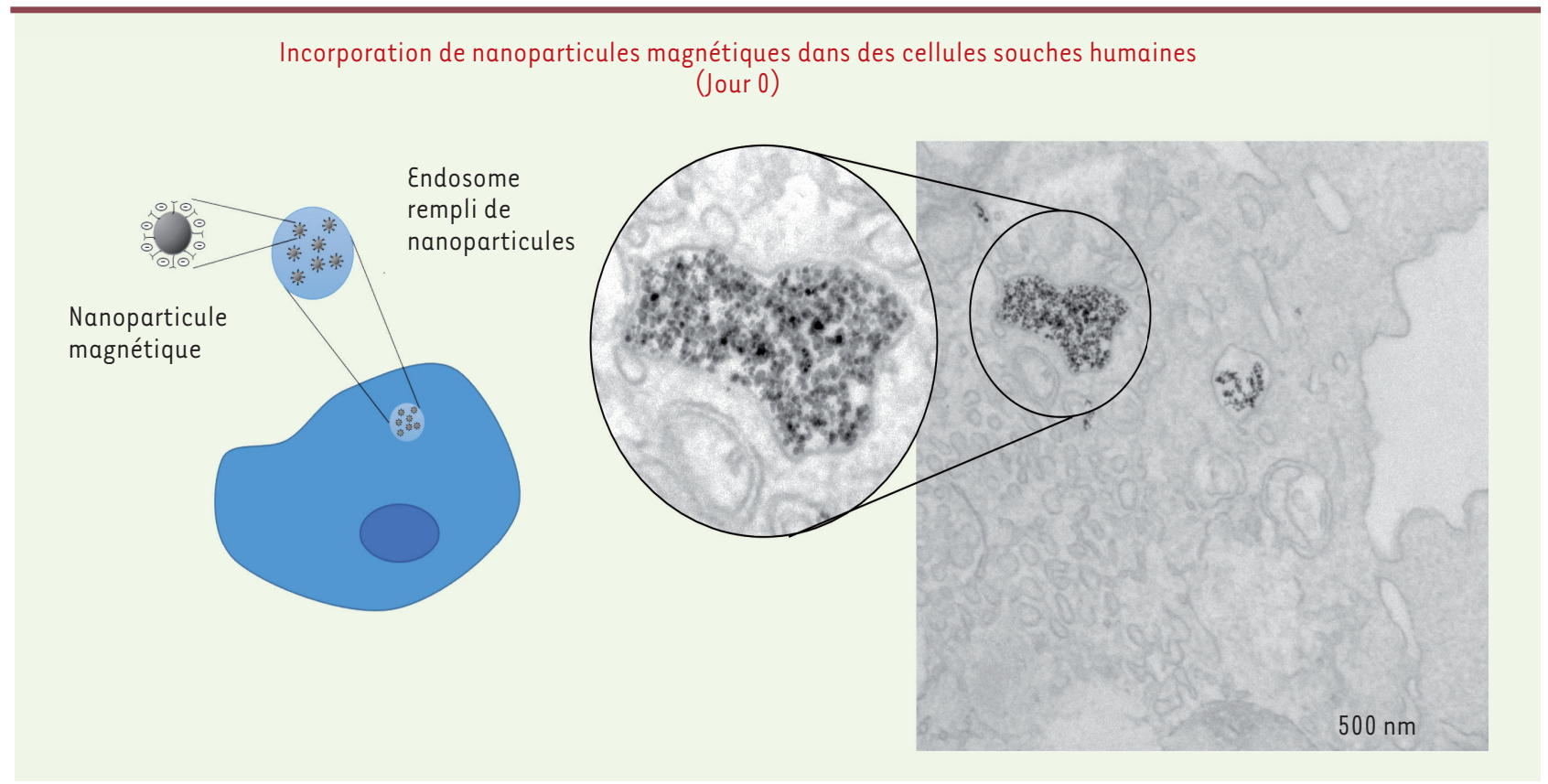

Figure 1. Les nanoparticules magnétiques sont incorporées dans les cellules souches par endocytose, puis stockées dans les endosomes. L'imagerie en microscopie électronique à transmission permet l'observation directe des nanoparticules.

entraîne une perte de magnétisme des cellules les ayant incorporées. Le fer libéré est alors stocké sous forme de ferrihydrite (non magnétique) à l'intérieur de la ferritine, la protéine de stockage du fer (Figure 2B). En revanche, lorsque les cellules sont cultivées en monocouches (2D), qu'elles restent non différenciées ou qu'elles soient orientées vers d'autres voies de différenciation (adipogenèse, ostéogenèse), une «re-magnétisation » spectaculaire des cellules est observée: l'aimantation des cellules diminue, puis augmente de nouveau (Figure 2C). Les nanoparticules sont tout d'abord détruites, libérant dans la cellule leur fer, qui est alors utilisé pour une biosynthèse de nouvelles nanoparticules magnétiques. Cette biosynthèse se déroule dans les endosomes, et semble liée à l'activité de la sous-unité $\mathrm{H}$ de la ferritine, qui possède une activité ferroxydase. Les nanoparticules biosynthétisées apparaissent clairement en microscopie électronique (Figure 2D), tandis que la ferritine non-magnétique observée précédemment devient difficilement détectable.
Une preuve de la possibilité d'une synthèse biogénique de nanoparticules magnétiques par des cellules humaines La présence de nanoparticules magnétiques a été observée dans divers organismes vivants tels que la truite arc-enciel, le pigeon voyageur, ou encore les bactéries magnétotactiques. Chez ces bactéries, différentes protéines spécifiquement dédiées à la synthèse des nanoparticules magnétiques ont pu être identifiées [6]. La présence de nanoparticules magnétiques a également été observée dans des cellules humaines [7], mais l'origine biogénique de ces nanoparticules n'avait pas été démontrée jusqu'à présent. Dans l'étude que nous avons menée, la «re-magnétisation » des cellules ne peut être expliquée autrement que par une synthèse de nanoparticules in situ. Le rôle de ces nanoparticules n'est cependant pas encore élucidé. En effet, la présence de nanoparticules magnétiques a parfois été liée à des maladies neurodégénératives, telles que les maladies d'Alzheimer et de Parkinson [8]. Ces résultats sont cependant contradictoires avec certaines études montrant la présence d'une quantité similaire de nanoparticules magnétiques chez des individus présentant ces maladies et chez des sujets témoins en bonne santé. Ce qui est confirmé, en revanche, c'est le dérèglement de l'homéostasie du fer corrélé à ces maladies et l'augmentation de la quantité globale de fer sous ses différentes formes de stockage [9]. Dans notre étude, lorsque nous observons la biosynthèse de nanoparticules magnétiques, la capacité des cellules souches à se différencier n'est pas affectée, même en présence d'une grande quantité intracellulaire de fer. Nous faisons donc l'hypothèse que cette néo-synthèse sert à préserver les cellules d'un excès de fer toxique : le stockage sous forme de nanoparticules magnétiques permettrait de limiter la quantité de fer libre et la production d'espèces oxydantes (liée à la réaction de Fenton) ${ }^{1}$ pouvant endommager les cellules. $\diamond$

Human stem cells can neo-biosynthesize magnetic nanoparticles after degrading man-made nanoparticles

\footnotetext{
La réaction de Fenton est une réaction d'oxydation avancée qui consiste à amorcer des réactions de décomposition du peroxyde d'hydrogène $\left(\mathrm{H}_{2} \mathrm{O}_{2}\right)$ par des sels métalliques (ions ferreux) afin de produire des espèces chimiques radicalaires $\left(\mathrm{HO} \cdot \mathrm{HO}_{2}\right.$, etc.), qui sont très réactives.
} 
Modèle sphéroïde (3D, chondrogenèse) : dégradation massive des nanoparticules

A Aimantation des cellules (pemu)

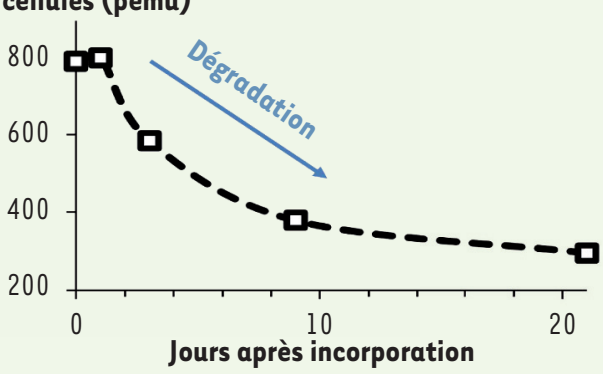

B

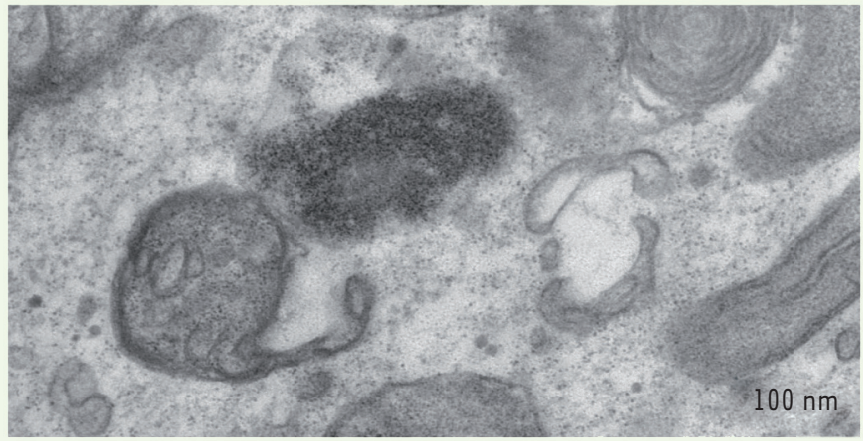

Modèle monocouche (2D) : re-magnétisation et néo-biosynthèse
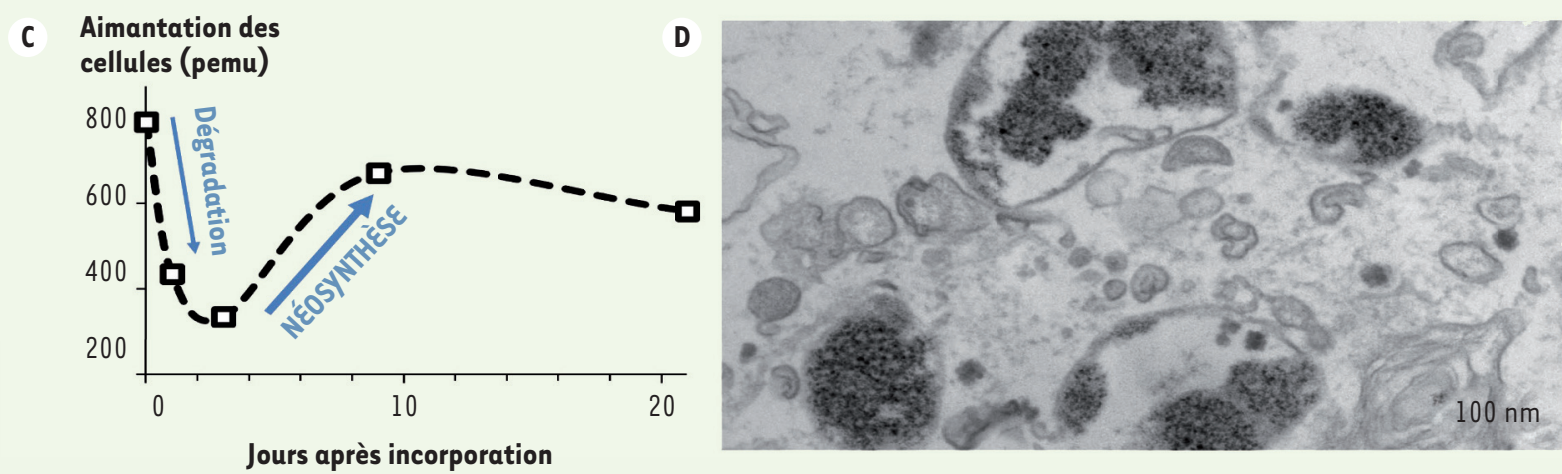

Figure 2. Suivi magnétique et par imagerie des nanoparticules dans les cellules souches. Après incorporation des nanoparticules, les cellules souches sont cultivées pendant 21 jours sous forme de sphéroïdes 3D pour induire la chondrogenèse ( $A$ et $B$ ), ou bien en monocouches dans des conditions de culture permettant une différenciation cellulaire (ostéogenèse et adipogenèse), ou le maintien dans un état non différencié ( $C$, $D$ ). La mesure du magnétisme des cellules par aimantation, exprimée en pemu $\left(10^{-12} \mathrm{emu}\right)(A, C)$, permet d'évaluer la transformation des nanoparticules. Une valeur de magnétisme décroissante indique une dégradation des nanoparticules, alors qu'une valeur croissante indique une synthèse de nouvelles nanoparticules. Dans le cas de la dégradation, le fer libéré est stocké dans la ferritine sous forme non-magnétique (ferrihydrite), comme on peut l'observer sur les images de microscopie électronique en (B) : petits spots de diamètre 5-7 nm, peu denses, localisés à la fois dans les endosomes et dans le cytoplasme. Dans le cas de la néosynthèse, les nanoparticules biosynthétiques produites in situ, au sein des endosomes (D), ont une structure cristalline d'oxyde de fer (maghémite ou magnétite) et apparaissent plus denses aux électrons.

\section{REMERCIEMENTS}

Nous remercions tous les co-auteurs de la publication originale [1]. Ces travaux ont été financés par l'union européenne (projet MaTissE 648779, $\varepsilon R(-2014-$ CoG)

\section{LIENS D'INTÉRÊT}

Les auteurs déclarent n'avoir aucun lien d'intérêt concernant les données publiées dans cet article.

\section{RÉFÉRENCES}

1. Van de Walle A, Plan Sangnier A, Abou-Hassan A, et al. Biosynthesis of magnetic nanoparticles from nano-degradation products revealed in human stem cells. Proc Natl Acad Sci USA 2019 ; 116 : 4044-53.

2. Ahrens ET, Bulte JW. Tracking immune cells in vivo using magnetic resonance imaging. Nat Rev Immunol $2013 ; 13: 755$.
3. Sangnier AP, Preveral S, Curcio A, et al. targeted thermal therapy with genetically engineered magnetite magnetosomes@RGD: Photothermia is far more efficient than magnetic hyperthermia. J Control Release 2018 ; 279 : 271-81.

4. Cazares-Cortes $\varepsilon$, Cabana S, Boitard C, et al. Recent insights in magnetic hyperthermia: From the "hotspot" effect for local delivery to combined magnetophoto-thermia using magneto-plasmonic hybrids. Adv Drug Deliv Rev 2019 ; 138 : 233-46.

5. Du V, Luciani N, Richard S, et al. A 3D magnetic tissue stretcher for remote mechanical control of embryonic stem cell differentiation. Nat Comm 2017 ; $8: 400$.

6. Schüler D. Genetics and cell biology of magnetosome formation in magnetotactic bacteria. FEMS Microbiol Rev 2008 ; 32 : 654-72.

7. Kirschvink JL, Kobayashi-Kirschvink A, Woodford BJ. Magnetite biomineralization in the human brain. Proc Natl Acad Sci USA $1992 ; 89$ : 7683-7.

8. Dobson J. Nanoscale biogenic iron oxides and neurodegenerative disease. FEBS Lett $2001 ; 496: 1-5$.
9. Belaidi AA, Bush Al. Iron neurochemistry in Alzheimer's disease and Parkinson's disease: targets for therapeutics. J Neurochem 2016; 139 : 179-97.
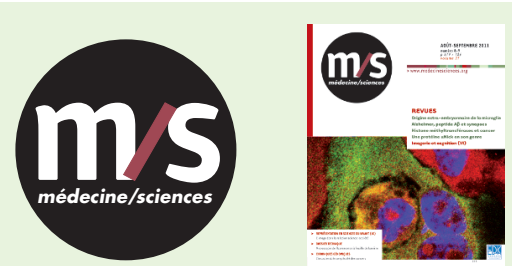

Abonnez-vous à médecine/sciences

Bulletin d'abonnement page 810 dans ce numéro de $\mathrm{m} / \mathrm{s}$ 